\title{
MITRAL AND TRICUSPID VALVE INNERVATION
}

BY

\author{
T. H. WILLIAMS
}

From the Department of Anatomy, The University, Manchester, 13

Received June 19, 1963

An aspect of cardiological anatomy which, though intriguing, has suffered sustained neglect, is that relating to heart valve innervation. In particular, no special neurological study of the atrioventricular valves appears to have been undertaken, presumably because this subject has not been recognized as a matter of importance. The cardiac valves are apparently regarded as structures that are mechanically passive and neurologically insignificant. However, the tricuspid and mitral valves are situated at two of the most strategic and vulnerable points in the circulatory system, being the sole entrances to the ventricular pumps from the pulmonary and peripheral vascular beds respectively: it would be imprudent, therefore, to ignore any aspect of the study of these valves that might disclose an unrecognized role for the cusps. Furthermore, since the mitral valve in particular is frequently involved in disease processes, the practical importance of acquainting ourselves as fully as possible about the atrio-ventricular valves is apparent. There is a need to understand more clearly the effects that valve pathology might have on cardiac function, apart from those resulting directly from distortion of the orifice. In addition, a complete appraisal of the effects of operative procedures carried out on the valves is not possible without a knowledge of the neural elements likely to be disturbed.

It was therefore decided to study in detail the nerve plexuses in the atrio-ventricular valves. Examination of the nervous elements present in normal valves was also an essential preliminary to degeneration experiments, designed to determine the source or sources of the nerve fibres. A great deal of work has previously been carried out to determine the distribution of parasympathetic efferent fibres to the cardiac musculature and coronary vessels, but the intracardiac afferent components contributing to the various cardiac reflex arcs have not been clearly defined. The study of heart valve innervation will facilitate the more comprehensive task of compiling accounts of entire reflex arcs, as a basis for understanding their function.

In the earliest accounts of the subendocardial nerve net (Kölliker 1852; Toldt, 1877), the cardiac valves received no attention. In the course of later investigations, the presence of nerve fibres in these valves was sometimes mentioned, but the extent of their innervation was neither explored nor explained. Smirnow (1895), while examining nerves and their endings in the mammalian endocardium, encountered nerve filaments that formed nets in the heart valves: these filaments, he believed, possessed no distinguishing features. In Michailow's study of the horse heart (1908), the cardiac valves received only a cursory note: during his scrutiny of the subendocardial plexus, which he described as forming an expansive net continuous throughout atria and ventricles, he found nerve fibres passing, as an extension of this net, into the valve cusps. Woollard (1926) described the innervation of the atrio-ventricular valves in conjunction with that of the subepicardial and endocardial tissues. In his account, the presence of abundant fine nerve plexuses was common to all these sites. The plexuses were composed of the finest fibres which usually gave off little branches that grew finer and finer until they disappeared. Following bilateral removal of the stellate ganglia of cats, Woollard found no diminution in the richness of these plexuses. The same 
author observed fine brush endings, but did not state categorically that these had been seen in the atrio-ventricular valves. Nettleship (1936), in his account of the subendocardial plexus in the cat, described arborizing and simple dot endings in the mitral and tricuspid valves. However, no illustrations of nervous elements within these valves were provided either by Nettleship or by previous workers. Lipp (1951), using a methylene blue staining technique, restricted his attention to the pulmonary valve of the guinea-pig, describing in particular the morphology of the rich nervous plexus that he found in the left cusp of this valve. Landau (1950) reckoned the endocardium near and over the cardiac valves among the sites where the nerve net was especially rich. Putting forward his conviction that the subendocardial plexus consists of a closed network of the so-called autonomic interstitial cells of Cajal, Meyling (1953) remarked that Terpstra (1943), in a study on the pathology of bacterial endocarditis, had observed the same nervous network in the atrio-ventricular valves.

\section{Materials AND MethodS}

Atrio-ventricular valves from guinea-pigs were first studied, the cusps from young adults of this species having the advantage of being of such a size that the valves may be readily dissected free, and being reasonably thin and transparent for microscopical investigations. Three other species were subsequently studied, namely cat, hedgehog, and rabbit, in order to establish whether a rich valvular innervation was a regular mammalian feature. By scrutinizing different species we could also observe details of the innervation patterns that were not readily apparent in the valves of the guinea-pig. With the exception of one large female guinea-pig, which will be referred to later, none of the animals was old or ailing.

Whole mount techniques were invariably employed, so as to preserve the structural continuity of the plexuses. The chief staining method used was a modification by Coupland and Holmes (1957) of the procedure developed by Koelle and Friedenwald (1949) for localizing cholinesterases. Other specimens were stained with an osmium tetroxide complex. Reticulin was also demonstrated, using Gordon and Sweets' (1936) silver method, to compare the appearances of these non-nervous fibres.

For localizing cholinesterases, the hearts were removed as soon as possible after death, the ventricles opened widely, and the tissues rinsed with cold isotonic saline. The valves were then carefully infiltrated, from the regions of their attached margins, with a solution of 1500 i.u. hyaluronidase in $5 \mathrm{ml}$. isotonic saline (Williams, 1962a). The purpose of the hyaluronidase was to facilitate the entry of both the fixative and the incubating fluid: the valves contain mucoid material that may represent remnants of cushion tissue. After 15 minutes at room temperature, the valve cusps were dissected free into cold isotonic saline. Some of the specimens were then fixed for two hours in ice-cold formol-saline, which had been stood over excess calcium carbonate. Since it has been said that unfixed tissues ought to be used for histochemical studies of enzyme distribution (Lewis, 1961), other cusps were stained before fixation in alcohol. Having been attached and dried on to clean slides, the valves were incubated for 8-16 hours at $30^{\circ} \mathrm{C}$. in the incubating medium recommended by Coupland and Holmes (1957) in their modification of the thiocholine technique, using either the acetyl or butyl substrate, and a $p \mathrm{H}$ of 4.9 for all species. The slides were then transferred to cold de-ionized water for 10 minutes, followed by dilute ammonium solution until no further darkening could be observed. After rinsing in water, three changes of alcohol ensured removal of ammonium sulphide and dehydration of the valves, as well as fixing the fresh specimens. Some of the preparations were at this stage counterstained with undiluted Leishman's solution, dropped briefly from a pipette. The procedure was completed by clearing in tetrachlorethylene and mounting in Canada balsam which had been dissolved in this solvent (R. E. Coupland, 1959, personal communication).

An osmium tetroxide -zinc iodide complex (Champy, 1959) was used to stain myelinated and unmyelinated fibres in atrio-ventricular valves from guinea-pigs. A 3 per cent solution of zinc iodide was prepared and brought to a $p \mathrm{H}$ of approximately 3.0 with $\mathrm{N}$ hydrochloric acid; four parts of this solution were mixed with one part of 1 per cent osmium tetroxide solution. The fresh tissues were then placed in the solution for 16 hours, at room temperature, in darkness. De-ionized water and clean glassware were used throughout. Acidification of the staining solution was carried out on the grounds that a low $p \mathrm{H}$ would reduce the tendency of the complex to dissociate, and would therefore reduce the amount of non-specific staining. After 16 hours, the valve cusps were washed in tapwater, placed on slides and, without being allowed to dry, dehydrated in alcohol, cleared in xylol, and mounted in 'deepex' mounting medium (Gurr).

Also for comparison, the valves from one guinea-pig were processed by a modified Bodian technique (Williams, 1962b, c). 
In two control guinea-pigs, a 10 per cent solution of chinese ink in isotonic saline was injected into the ascending aorta at death, with the aorta clamped beyond the injection site. After fixation in cold formolsaline, the valves were dissected free. The valves from one specimen were then processed for acetylcholinesterase localization, but the other injected valves were mounted unstained.

\section{RESULTS}

In these observations, attention is restricted to the neural elements within the atrio-ventricular valves of guinea-pigs and other animals, and the further course of the nerve fibres outside the limits of the valve cusps is not described here.

In each of the species studied, cholinesterase-positive filaments form networks with fine meshes which are common to every cusp (Fig. 1A). The plexuses are so profuse that the possibility of non-nervous elements contributing towards the appearances was considered. A number of control preparations was therefore studied. In valve cusps from guinea-pigs and cats, stained for reticulin by the Gordon and Sweets method, the distribution, dimensions, and configuration of the fibres were examined and compared with the cholinesterase-positive filaments. Covering large parts of the cusps, dense and tangled arrangements of reticulin fibres are seen, and they are so numerous in some areas as to obscure almost all trace of other elements. The most noticeable feature is the arrangement of the fibres after a general fashion along the lines of stress, running chiefly along the chordæ tendineæ and from the attachments of chordæ towards the bases of the cusps. No branching of the reticulin fibres is seen in this tissue: they form spirals or pursue irregular wavy courses and often appear to end like pulled-out threads. From these points of difference it is possible to assert that the cholinesterase-positive elements are fibres of an entirely different character.

The general appearance of the cholinesterase-positive network, when seen under low magnification, bears some resemblance to vascular networks which in some species and situations possess this enzyme. The possibility that some of the strands might be blood vessels was investigated by counterstaining cholinesterase preparations of valve cusps so as to demonstrate the nuclei of endothelial cells. None were found in the vicinity of the cholinergic fibres, and phase contrast microscopy also failed to disclose any relation between blood vessels and the enzyme-rich network. Since the specimens that had been perfused through the coronary vessels with chinese ink showed that the vascular channels, which seldom extended far from the base of a cusp, did not exhibit enzyme activity in their walls, it was concluded that the cholinesterase-positive strands were all nervous in nature.

Guinea-pig. Fig. 1A shows a cusp from a tricuspid valve, stained to localize acetylcholinesterase activity. In this as in other specimens, nerve bundles could always be followed in from the attached border of the valve. Extending across the entire cusp, from the base to the free margin, is a rich skein of nerve fibres, the individual threads of which are distinguished by the presence of closely-set, enzyme-rich beads. These presumably indicate the sites, within the nerve fibres, of organelles containing acetylcholinesterase (AChE). The bundles of the plexus split up repeatedly, the branches often linking up with others. As the smaller nerve fascicles ramify in the spaces between the larger bundles, filaments in ones and twos leave them to pursue an irregular course, often crossing many others in their path. In preparations showing good localization, the discrete nerve fibres, isolated or travelling in bundles, can be distinguished (Fig. 1B). Individual fibres branch dichotomously, giving rise to very fine beaded filaments. Organized nerve endings are not visible in the cusps of the mitral or tricuspid valves of guinea-pigs, although the cholinesterase-positive portion of a fine fibre is frequently seen to come to an abrupt end. Arrows in Fig. 1A indicate filaments which show this appearance.

In the valvular plexus of the guinea-pig, numerous bundles and filaments of the kinds described can be followed in every direction, but the general tendency is for them to proceed towards the free margin of each cusp. Close to the attached margin or base, traces of cardiac muscle are sometimes found, particularly in the mitral valve. When this muscular component is present in guinea-pig valves, it possesses the staining characteristics of atrial rather than ventricular muscle. In this species 
FIG. 1.-(A) Rich nerve plexus in a guinea-pig tricuspid valve, stained by the thiocholine method. Arrows indicate presumed endings. $(\times 100$.) (B) A portion of the same field at higher magnification, to demonstrate the beaded nerve fibre. $(\times 300$.) (C) Single heavily myelinated fibre, accompanied by many fine beaded filaments, in guinea-pig mitral valve (osmium preparation). ( $\times 150$.) (D) Nerve fibres in a chorda tendinea of guinea-pig tricuspid valve. Cusp attachment above (thiocholine preparation). $(\times 80$.

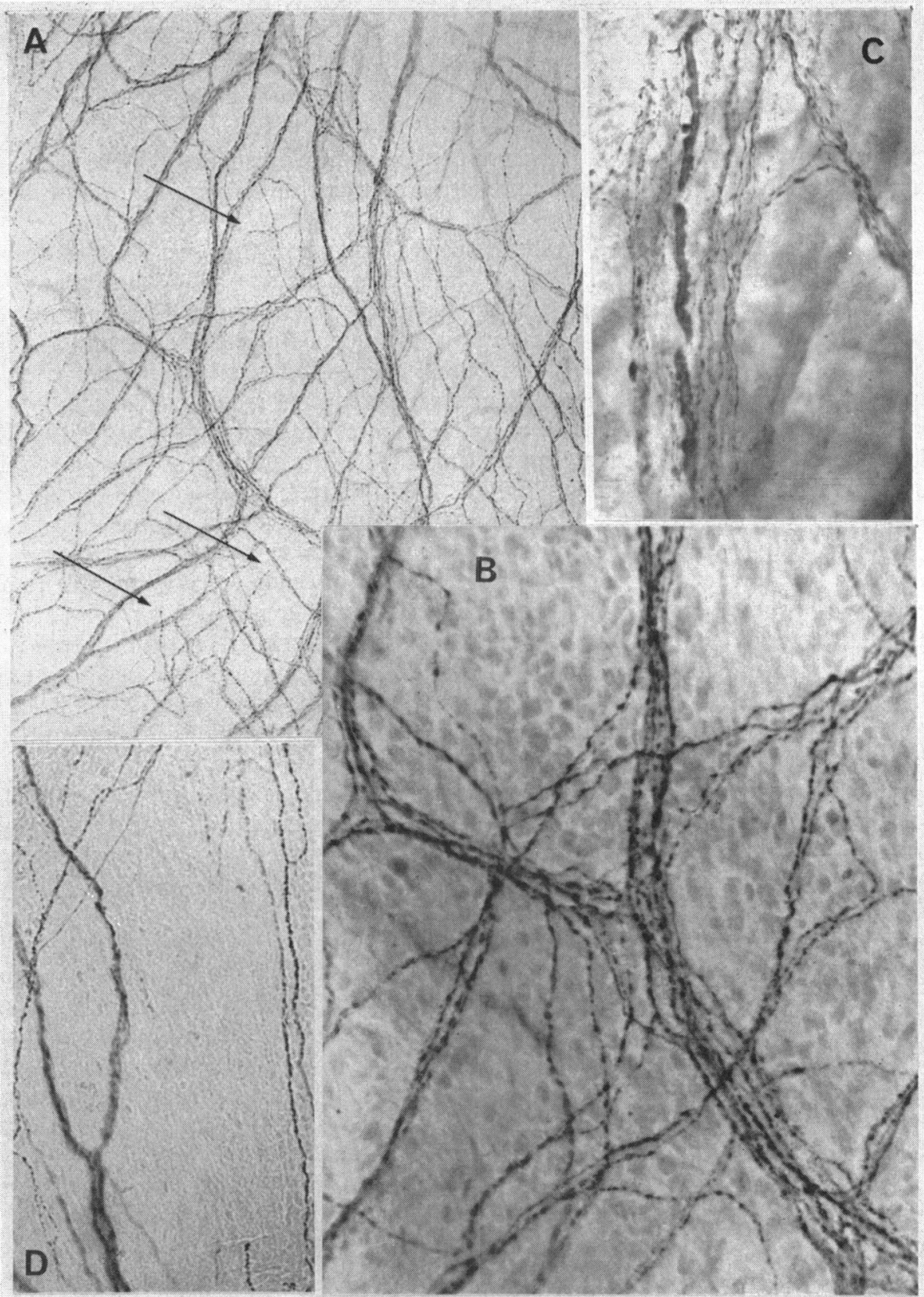


the atrial muscle gives a stronger cytoplasmic reaction for $\mathrm{AChE}$ than the ventricular muscle: furthermore, atrial muscle fibres possess a rich cholinergic network, which consistently shows greater $\mathrm{AChE}$ activity than does the network pervading the ventricular myocardium.

Towards the free margin of a cusp, the nerve plexus gradually becomes finer as the thicker bundles branch progressively. As a rule, the plexus remains rich right down to the edge of the cusp, although here it is composed of very fine strands of nerve fibres and single filaments. Individual variation is apparent, however, in the density of the network in this part of the cusp, and in specimens that show the least concentrated plexuses, fine filaments can often be followed to what seem to be their terminations. At the junctions between valve cusps and chordæ tendinex, nerve strands in considerable numbers are often seen passing between the two structures (Fig. 1D). It was not possible to determine whether the fibres passed upwards from the chorda or downwards from the cusp: indeed, some of the strands and individual nerve fibres appear to split dichotomously and to pass in both directions (Fig. 3C).

Infrequently in the guinea-pig specimens, oval, round, or less often irregular, swellings are found lying within or against nerve strands. These swellings are much larger than the beads which are consistently present along the course of the nerve fibres. A few of them are more than $20 \mu$ across. They possess definite, smooth outlines and stain darkly and homogeneously for AChE, although the intensity of the enzyme reaction appears to vary from one swelling to another. Of the 12 guinea-pigs in this series, only 4 exhibited this phenomenon in one or both atrio-ventricular valves, and only one animal, a very large fat aged female, showed AChE-positive bodies of this kind in both valves. In the valves from this animal only, a few examples of nerve fibres showing signs of fragmentation were also seen, and swellings of the kind described above were found on one or two of these fibres. This guinea-pig, which had originally been intended for operative work, died suddenly before the operation was performed, after receiving only an anæsthetic dose of "nembutal". This specimen excepted, none of the preparations scrutinized, amounting to 22 atrio-ventricular valves from 11 young animals, showed fragmented nerve fibres, and of the three valves that showed cholinesterase-positive swellings no more than two fibres in each possessed these enlargements.

Occasionally, a bundle of beaded nerve fibres appears on close examination to contain a nerve fibre different in type from the others. After making allowances for possible variations, it is concluded that this fibre is undoubtedly very much thicker in calibre than any of the beaded filaments, but its relatively low activity precludes a detailed study of its structure by the cholinesterase technique. However, it is certain that some, at least, of the coarse fibres pass into chordæ tendinex. At this stage it may be recalled that myelinated sensory fibres in other situations fail to stain satisfactorily with the thiocholine method, especially near their terminations (Williams and Dixon, 1963). A study was, therefore, made of guinea-pig mitral valves, stained by an osmium tetroxide complex, and these show clearly that each valve contains one or two heavily myelinated nerve fibres, each being accompanied across the cusp by many beaded filaments (Fig. 1C). Each myelinated fibre can be followed across a cusp and into a chorda without any obvious change in calibre being seen, but the mode of termination of these fibres was not ascertainable in the osmium preparations.

Cat. In mitral and tricuspid valves of cats and kittens, stained by the thiocholine technique, the characteristics of the neural elements present were compared with the guinea-pig preparations, and the salient features were noted. A nervous plexus, giving a fairly strong reaction for AChE, was displayed in each valve, and an inconstant finding in the cat, as in the guinea-pig, is the presence, in the vicinity of the attached margin, of cardiac muscle which possesses the heavy staining property and rich cholinergic innervation that are peculiar to the atrial myocardium.

When viewed under low magnification the nervous network in each cusp appears to be less dense than that already described in the guinea-pig, but it becomes apparent under high magnification that the interstices of the network are occupied by large numbers of very fine filaments, the most slender of which are beaded and pursue an irregular course, often crossing strands of the main plexus. Beading of individual nerve fibres was not observed in the coarse strands that passed into each cusp to enter the plexus. 
Fig. 2.-Cat atrio-ventricular valves stained by the thiocholine method. (A) Arborizing nerve strand entering from attached margin of cusp (above). $(\times 50$.$) (B) Single arborization$ showing numerous cholinesterase-positive $(\mathrm{AChE})$ swellings. $(\times 130$.) (C) Portion of the same field at higher magnification, showing presumed degenerative vesicles and beaded nerve fibres. $(\times 430$.) (D) Similar swellings in a cat valve which showed a plexiform innervation pattern. $(\times 500$. 
When the valve cusps that possess this plexiform innervation are examined, the complexity of the main network, taken together with the fine filaments in the interstices, is such that the course and distribution of an individual nerve strand cannot be followed for more than a very short distance. This type of plexus, having at first sight the appearance of a closed net, is not present throughout all portions of cat valves.

Only in situations where the plexus is less complex can the finer neural elements be studied closely. The nerve fibres demonstrated in a cusp often fail to reach the free margin, and in such specimens many of the smaller nerve fascicles that lie at the periphery of the plexus can be traced with certainty. These outlying fibres arborize as they proceed across the cusp, their finest divisions having a beaded appearance.

Another appearance enables one to ascertain, without any difficulty, the mode of termination of nerve fibres in cat valves. In some valves, a number of the incoming nerve strands are located at wider intervals and their distributions do not overlap, so that it is possible to follow and observe the ramifications of a single strand. Several such examples were seen (Fig. 2A), although this arrangement occurs as an exception to the rule. In such cases the main strand sometimes gives off, close to the attached margin of the cusp, a lateral branch which travels for some distance parallel to this margin before ending as a few terminal twigs which pass into the cusp (Fig. 2A). Other main nerve stems give rise to lateral branches only after passing some distance into the cusp: these lateral branches then form terminal aborizations of varying complexity and covering areas of different size. At any point between the attached margin and the central region of a cusp, the main bundles begin to break up into their terminal branches, bifurcating repeatedly without the branches rejoining, after the manner of terminal arborizations. The fine threads derived from the main strands by the process of repeated branching differ from the thicker nervous elements in being studded with small closely arranged centres of AChE activity, which confer a beaded character upon the filaments. These filaments vary greatly in length, but shorter ones predominate and give off, along their course and at their terminations, short beaded twigs that often have a spiky appearance. The twigs end abruptly, and when they are examined by phase contrast it appears that the ends of the stained portions correspond with the actual terminations of the filaments.

Many such formations were examined without finding a single ending of a more complex variety than the beaded threads. However, in one arborization seen in the tricuspid valve of an adult cat, several round or oval bodies stood out prominently on account of their high AChE activity (Fig. 2B and C): they lay along the course of nerve fibres and were several times larger than the enzyme-rich beads described as being present in fine nerve filaments. This finding resulted in a search for similar entities in other cat preparations. In a few cusps only, AChE-positive bodies were observed along the strands or at the junctions of the plexus (Fig 2D).

The feline atrio-ventricular valves are sufficiently large to enable one to distinguish the commissures, or minor cusps, which are interposed between the major cusps of the valves. Numerous fine nerve strands pass into these commissures from the region of the attached margin. In contrast to the majority of strands which pass into the major cusps, the fibres in the commissures show few bifurcations and no great tendency to arborize or to form complex networks.

Nerve fibres can be followed into the chordæ tendineæ from the cusps in cats as in guinea-pigs. In the cat, thick but relatively pale and unbranching strands occasionally extend from the attached margin of a cusp and, after leaving the cusp, continue along the length of a chorda to reach the papillary muscle (Fig. 3A). Many fine nerve filaments can also be traced into the chordæ, and an isolated, darkly staining nerve fibre sometimes runs through the substance of a muscular chorda between the cusp and the papillary muscle (Fig. 3B).

Rabbit and Hedgehog. The mitral and tricuspid valves of these animals likewise present such a complex innervation pattern that, for the sake of simplicity, attention will be directed only to the chief points that characterize the valvular neural elements in these species. In hedgehog atrioventricular valves, the plexus of fine nerves is very rich and an arborization pattern is not apparent. As in the cat, the nerves in rabbit valves exhibit a plexiform arrangement or alternatively take the 


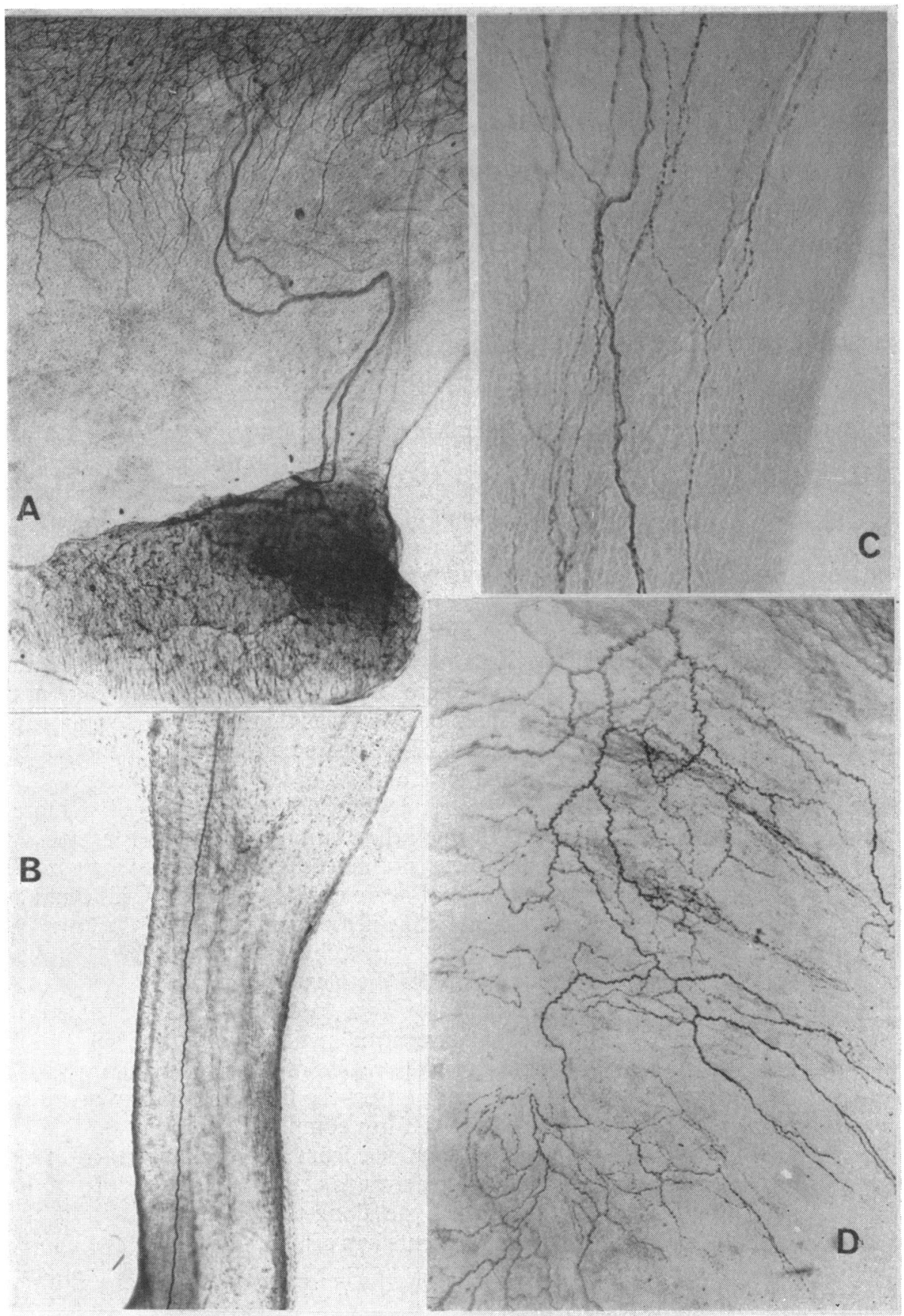

Fig. 3.-Thiocholine preparations. (A) Cat mitral valve showing two unbranching coarse fibres passing between attached margin (above) to chorda tendinea (below). ( $\times 65$. (B) Nerve fibre passing through muscular chorda tendinea of cat mitral valve. $(\times 65$.) (C) Nerve fibres passing through guinea-pig chorda tendinea. $(\times 80$.$) (D) Arborization$ pattern in rabbit mitral valve. $(\times 85$.) 
form of strands that run down from the region of the attached margin to arborize without overlap in the cusp (Tig. 3D). Although these strands are somewhat closely situated to each other, their distribution without overlap occurs more frequently in the rabbit preparations than in cat valves. In the rabbit cusps, the finest filaments visualized present a beaded appearance, the closely set "beads" marking the sites of AChE activity. The terminal portions of these filaments sometimes show a tendency to run parallel to one another.

\section{Discussion}

The thiocholine technique has proved useful for displaying autonomic innervation patterns and in the present investigation this histochemical method for localizing cholinesterases has been utilized solely as a neurohistological tool for demonstrating the neural elements in heart valves.

Judging from the morphological observations made on the valvular plexuses with the aid of this technique, in combination with perfusion of the coronary arteries with chinese ink or counterstaining of the tissue to show the endothelial nuclei, only a negligible proportion at most of nervous elements present were in any way related to the small vascular channels found close to the attached margin of each guinea-pig cusp. Therefore, only nerve fibres associated with the atrial muscle, which is sometimes present in the cusps, and possibly some of those that pass into the chordx tendinex, could be regarded as motor in function. The great majority of the nerves distributed across the cusps had neither muscular nor glandular elements to supply, and were considered to be sensory.

A well-developed neural apparatus was found in every cusp of the atrio-ventricular valves of all the animals studied, and this suggests that both mitral and tricuspid valves are special sensory areas or reflexogenic zones. It has been pointed out (Mitchell, 1956) that the subendocardial nerve networks are especially evident in the regions adjacent to the cardiac valves, and one believes that these concentrations of nerves are accounted for by the fibres that enter the valves to supply the cusps and the chordæ. The problem of tracing the sources of these cuspidal nerve fibres has been held over for a subsequent investigation.

While the function of a motor nerve fibre may generally be inferred from the histological relations of its terminations, in the case of sensory nerves sites of termination are less commonly indicative of precise function. Bearing in mind that any attempt to correlate structure and function of the sensory components in valves suffers from this difficulty, a purpose can be tentatively ascribed to these plexuses after studying their organization within the cusps. A prominent feature of the nervous plexuses in the atrio-ventricular valves of all species studied has been their widespread distribution throughout all the cusps. It seems unlikely, therefore, that the main network is concerned with the measurement of any bulk property of the blood-such as temperature or sodium concentration-since these properties, being virtually the same throughout each chamber, would require relatively small sensory zones for their evaluation. On the other hand, extensive plexuses such as have been described in the valves would be appropriate for the measurement of flow characteristics, namely the recording of blood movements across different portions of each cusp.

There is at present insufficient evidence for deciding which effector mechanisms are influenced by stimulation of the valvular network. Nevertheless it seems likely that the plexuses are a provision for precise control of the orifices themselves and of the papillary muscles, in accordance with local needs. During ventricular diastole, when blood is flowing through the atrio-ventricular valves, perfect control of the orifices would minimize the energy dissipated as frictional loss. Although the extent of this loss has not been ascertained, it is augmented and made more significant by the pulsatile nature of the flow and the turbulence in the fluid. During ventricular systole, rapid and accurate apposition of the valve cusps may be partly dependent upon a sensory mechanism within the cusps.

The thickly myelinated nerve fibres observed in guinea-pig mitral valves present a similar problem in correlation. Again it is impossible on the available evidence to evaluate their significance, but in this instance the dimensions of the axons provide a clue. On this basis it may be 
argued that they are designed to conduct signals rapidly to the central nervous system, and perhaps $\stackrel{\Xi}{\rightrightarrows}$ they form part of a timing device or are concerned in measuring intraventricular pressure. Although $c$. the function of the thick nerve fibres is at present hypothetical, they may be proprioceptive since they can be traced into the papillary muscles and chordæ tendineæ.

Whitteridge (1948), working on cats and a rabbit, detected impulses travelling up the vagus in time with the cardiac rhythm. Using a pen-recording electroencephalograph, this finding has been confirmed for the guinea-pig (unpublished observation). The signals taken from the guinea-pig vagus were in synchrony with ventricular systole, but no indication of the speed of nervous transmission was obtained, and the number of fibres participating was not assessed.

The cholinesterase-positive swellings found in relation to nerve fibres in some of the valve cusps are interesting. They have been observed in cat and guinea-pig atrio-ventricular valves, and in general they were situated along the course of nerve strands rather than terminally. The acetylcholinesterase activity of the swellings varied considerably, but more often than not they gave a fairly strong reaction. The absence of swellings from the majority of guinea-pig valves and their random occurrence in ones and twos in others leads one to think that in this species they indicate degenerative changes, particularly since they were a more prominent feature in the one animal that showed fragmented nerve fibres and that was senile. One finds support for this view in those cat preparations that presented discrete arborizing nerve formations: here a single arborization in the tricuspid valve of an adult cat showed numerous swellings (Fig. 2B), while other arborizations were entirely devoid of such structures. It is of some importance to recognize that these presumed degenerative phenomena occur predominantly in older animals, and, on account of the high probability that degenerating nervous elements may be found in ostensibly normal material from such animals, care should be taken in selecting only young healthy specimens for degeneration experiments. Otherwise it is less easy to sustain any claim to reliability.

The main object in describing the neural elements within the tricuspid and mitral valves has not been to show that a profusion of nerve fibres exists in these situations, but rather to explain the functional significance of these nervous elements. Previous anatomical references to the innervation of the atrio-ventricular valves have been to some extent undiscriminating, the valvular plexuses having been considered simply as extensions of the endocardial network. It is precisely because the morphology of these strategically placed nerves indicates special and probably important reflexogenic activities, that investigations to elucidate their extravalvular course by a degeneration method (Williams, 1963) are proceeding.

\section{SUMMARY}

Intrinsic nerves in mitral and tricuspid valves from guinea-pig, cat, rabbit, and hedgehog can be demonstrated by a cholinesterase method. They are described and illustrated with a view to suggesting clues to their functional significance.

In the guinea-pig, the atrio-ventricular valves contain nerve fibres of two distinct types: fine beaded fibres which form a rich network throughout all cusps; and scanty, heavily myelinated fibres, about $8 \mu$ in diameter, which are seen in the mitral valve and can be traced into chordx tendineæ. The latter type can be demonstrated more clearly using an osmium stain. The morphological features of these two nervous elements suggest that the rich plexus of fine fibres is concerned with measuring flow properties of the blood as the latter moves across the cusps, while the thick myelinated fibres may transmit impulses concerned with timing or proprioception.

In cat mitral valves also, very thick nervous strands can be traced into the chordæ. The plexuses within the cusps, particularly in older animals, are less dense than in guinea-pig valves, and evidence that the nerve strands arborize is presented, although the individual arborizations usually overlap, giving the appearance of a terminal network. This finding was confirmed in the rabbit; but the hedgehog, like the guinea-pig, possesses extremely rich plexuses, so that no distinct arborization pattern is visible. 
In guinea-pig and cat preparations, particularly those taken from older animals, acetylcholinesterase-positive swellings are occasionally seen along the course of nerve fibres: these are probably degenerative vesicles, and it is stressed that young healthy animals should therefore be selected for nerve-degeneration experiments.

I wish to thank Professor G. A. G. Mitchell for his encouragement and for his detailed criticism of the manuscript, and Mr. P. Howarth for the photomicrography.

\section{REFERENCES}

Champy, C. (1959). Modifications de la technique de Champy au tétraoxide d'osmium-iodure de potassium. Résultats de son application à l'étude des fibres nerveuses. C.R. Soc. Biol. (Paris), 153, 939.

Coupland, R. E., and Holmes R. L. (1957). The use of cholinesterase techniques for the demonstration of peripheral nervous structures. Quart. J. micr. Sci., 98, 327.

Gordon, H., and Sweets, H. H., Jr. (1936). A simple method for the silver impregnation of reticulum. Amer. J. Path., 12, 545.

Koelle, G. B., and Friedenwald, J. S. (1949). A histochemical method for localizing cholinesterase activity. Proc. Soc. exp. Biol. (N.Y.), 70, 617.

Kölliker, A. (1852). Handbuch der Gewebelehre des Menschen, Vol. 2, p. 539. Engelmann, Leipzig.

Landau, E. (1950). Contribution à l'innervation du coeur (fibres myélinisées). Acta anat. (Basel), 9, 227.

Lewis, P. R. (1961). The effect of varying the conditions in the Koelle technique. In Histochemistry of Cholinesterase, Symposium, Basel, 1960. Bibl. anat. (Basel), 2, 11.

Lipp, W. (1951). Studien zur Herzinnervation. I. Die Innervation der Pulmonalisklappen. Acta anat. (Basel), 13, 30.

Meyling, H. A. (1953). Structure and significance of the peripheral extension of the autonomic nervous system. J. comp. Neurol., $99,495$.

Michailow, S. (1908). 'Die Nerven des Endocardiums. Anat. Anz., 32, 87.

Mitchell, G. A. G. (1956). Cardiovascular Innervation. Livingstone, Edinburgh and London.

Nettleship, W. A. (1936). Experimental studies on the afferent innervation of the cat's heart. J. comp. Neurol., 64, 115 .

Smirnow, A. (1895). Ueber die sensiblen Nervenendigungen im Herzen bei Amphibien und Säugetieren. Anat. Anz., 10, 737.

Terpstra, J. I. (1943). Over de pathogenese van de bacteriele endocarditis. Thesis, Utrecht.

Toldt, C. (1877). Lehrbuch der Gewebelehre, p. 245. Enke, Stuttgart.

Whitteridge, D. (1948). Afferent nerve fibres from the heart and lungs in the cervical vagus. J. Physiol. (Lond.), $107,496$.

Williams, T. (1962a). "Spreading factor", used before fixation, as an aid to cholinesterase localisation. J. Histochem. Cytochem., 10, 435.

(1962b). The staining of nervous elements by the Bodian method. I. The influence of factors preceding impregnation. Quart. J. micr. Sci., 103, 155.

(1962c). The staining of nervous elments by the Bodian method. II. The effects of some variable factors at the impregnation stage. Quart.J. micr. sci., 103, 297.

(1963). Terminal axonal degeneration visualized by a cholinesterase method. J. Anat. (Lond.), In the press.
, and Dixon, A. D. (1963). The intrinsic innervation of the soft palate. J. Anat. (Lond.), 97, 259.

Woollard, H. H. (1926). The innervation of the heart. J. Anat. (Lond.), 60, 345. 